\title{
Backbone Source and Positional Broadcast Routing for Emergency System in Urban VANETs
}

\author{
Vansh Kaushal \\ Student, Bharati Vidyapeeth's \\ College of Engineering, New \\ Delhi, India
}

\author{
Venuprea \\ Student, Bharati Vidyapeeth's \\ College of Engineering, New \\ Delhi, India
}

\author{
Surjeet \\ Associate Professor, Bharati \\ Vidyapeeth's College of \\ Engineering, New Delhi, India
}

\begin{abstract}
Disseminating warning information by Vehicular Ad hoc Networks (VANETs) is of great significance to alleviate traffic problems in time critical applications in future Intelligent Transportation Systems (ITS). In the urban express environment, it is critically challenging to design efficient dissemination mechanisms with strict Quality of Service $(\mathrm{Q} o \mathrm{~S})$ requirements due to complex road structures, severe channel contention, message redundancy etc. In this paper, the Backbone Source and Positional Broadcast Routing (BSPBR) protocol has been proposed to lower message transmission delay and increase reliability. It employs dynamically generated backbone nodes as source nodes based on movement and link quality between vehicles based on a fuzzy logic. Novel forwarding node selection scheme is followed in all propagating directions using iterative partition, mini-slot and black-burst exchange. A single node is successfully chosen using the backbone ranking. Bidirectional broadcast, multi-directional broadcast and directional broadcast are designed on the basis of the position of senders to enable emergency messages to cover the target area seamlessly. Theoretical analysis and simulation results are used to show significant improvement in throughput with marginal effects on end-to-end delay and packet delivery fraction by the proposed protocol over other existing alternatives.
\end{abstract}

\section{General Terms}

AODV, QoS, routing, VANETs

\section{Keywords}

Backbone node, data dissemination, directional broadcast, emergency message, QoS, VANETs

\section{INTRODUCTION}

Vehicular Ad Hoc Networks (VANETs) have been attracting interest due to their novel application to the Intelligent Transportation System (ITS). They primarily support Vehicleto-Vehicle (V2V) communication [1] at low cost by using a license-free spectrum and minimum infrastructure. They extend the communication coverage area by information exchange between nodes in a distributed manner. Data dissemination [2] is a promising application in VANETs for safety aid like broadcasting warning messages (e.g. accident, blocked street, traffic congestion etc.) that need to be delivered to the nearby vehicles. Efficient broadcasting [3] is required in cases when the transmission range is not enough to cover all intended receivers. Such kind of alert information assists drivers to make early driving decisions like alternate route determination to avoid traffic jams, slowing speeds in low visibility conditions or road clearance for emergency vehicles etc. Most of these value-added applications require a light weight and reliable multi-hop broadcast protocol as the network layer communication protocol. However, changing vehicular densities, mobility and limited bandwidth of wireless communication make it difficult to provide high packet dissemination ratio and low end-to-end delay. More specifically, three main issues [4] need to be seriously considered in the protocol design: (i) packet dissemination is low due to packet collisions and channel fading because of lack of any acknowledgement (ii) network congestion needs to be avoided by a light weight multi hop packet forwarding and retransmission mechanism (iii) large number of sender nodes contest for channel access at the same time increasing wait time. Since high latency can make a message out-of-date, problem of end-to-end delay needs to be addressed.

The simplest way to disseminate a message to the entire network is Flooding. However, an uncontrolled rebroadcast leads to high level of contention, packet collisions and eventual data loss [5]. This is called the Broadcast Storm problem. Protocols proposed to deal with this issue are classified into two categories [6] as: (i) sender-oriented (deterministic) protocols and (ii) receiver-oriented (nondeterministic) protocols. The receiver-oriented protocols use an autonomous approach to decide whether or not to forward a packet at each node upon reception. They use schemes like: weighted p-persistence, slotted 1-persistence and slotted ppersistence scheme. They do not entirely eliminate broadcast repetition. The sender-oriented protocols select the relay nodes and require acknowledgement from the receivers. They do not consider the channel fading and other factors in this selection and suffer high overhead and large collision probabilities.

Traditional Ad hoc broadcast protocols can hardly be applied to urban VANETs directly due to the diverse QoS requirements [7] of safety related services such as low latency, high reliability, low redundancy etc. Characteristics such as vehicle density, moving velocity, position etc. need to be taken into account to improve broadcast performance. In order to efficiently address the aforementioned challenging issues in urban VANETs, a Backbone Source and Positional Broadcast ( BSPBR) protocol has been proposed which takes into account vehicle movement dynamics, link quality and road layout of the of the transportation system. AODV based on periodic message interchange for updating of network status has been considered as the base comparative protocol. The contributions of the paper include:

- Backbone nodes are selected autonomously based on the hello message exchange by taking into account vehicular characteristics based on a fuzzy logic to identify as source nodes.

- Efficient forwarding node selection scheme is presented to quickly select a remote neighboring node using iterative partition, mini-slot and blackburst exchange. This greatly reduces emergency transmission delay. 
- Based on the forwarder node selection scheme, three broadcast strategies such as bi-directional broadcast, multi-directional broadcast and directional broadcast are designed to disseminate message. This ensures reliable and seamless coverage of target area.

The remaining paper is structured as follows: Section 2 gives a brief overview of the related work. After introducing the system model in Section 3, the details of the backbone source node identification and three broadcast strategies are illustrated in Section 4. A theoretical model is presented in Section 5 to evaluate performance of the proposed protocol in terms of throughput and end-to-end delay. Section 6 gives the simulation results and graphs followed by concluding remarks in Section 7.

\section{RELATED WORK}

Broadcasting in urban VANETs is highly complex due to varying road structures, severe channel contention, message redundancy etc. Many efforts have been devoted to devising efficient solutions to support safety applications recently. A street- based broadcast scheme is presented in [8] where each vehicle periodically broadcasts hello messages containing position information to its neighbours. In case of traffic accidents, the farthest node serves as the relaying node. [9] Proposes a cross-layer broadcast scheme for safety message dissemination. It divides them into prioritized categories for periodic exchange. In [10], a trinary partitioned black-burst based broadcast protocol is presented to support time-critical message dissemination. It selects a forwarding node using a mini-DIFS mechanism and iterative partitions of target area. The Cross Layer Broadcast Protocol (CLBP) [11] selects a forwarding node according to a novel metric considering distance, velocity and packet error rate. [12] Proposed a solution to the broadcast storm problem by using speed adaptive probabilistic flooding for varying vehicular density in traffic scenarios. [13] Introduced the combined concept of black-burst and multi-channel transmissions to deal with Clear to Broadcast (CTB) collisions and reduced propagation speed. Aforesaid approaches lack multi-directional broadcast support at intersections.

Urban environments are categorised by dynamic vehicular density, varying road structures, intersections and bidirectional paths. Some broadcast schemes are designed specifically for urban vehicular networks. In [14], an enhanced Street Broadcast Reduction (eSBR) scheme is presented to address the broadcast storm problem. It decides to rebroadcast a message if its distance to the sender is larger than the threshold. Profile-driven Adaptive Warning Dissemination Scheme (PAWDS) [15] utilizes eSBR to reduce dissemination based on vehicle density. Integrated protocols have been presented to alleviate message redundancy and reduce latency. In [16], Ad hoc Multi-hop Broadcast (AMB) and Urban Multi-hop Broadcast (UMB) are designed to address the issues of latency, reliability and broadcast storm. They utilize the directional broadcast to select forwarding nodes using Request to Broadcast (RTB)/ Clear to Broadcast (CTB) handshakes. Binary Partition Assisted Broadcast (BPAB) [17] uses different broadcast strategies according to position of emergency message senders. [18] Has confirmed the feasibility of a layout-aware emergency message handshake mechanism and redundant relay node adaptation mechanism to improve reliability with less delay. Most of the prevalent protocols result in inefficient multi-hop broadcasting when the number of data flows is large. This paper proposes a new approach of backbone source node and subsequent location based broadcasting to allow seamless coverage of large transmission range for time critical applications.

\section{SYSTEM MODEL}

BSPBR aims at emergency message broadcast in urban settlements with a large number of moving vehicles without the support of roadside infrastructure. Vehicles can move in opposing directions in multi-lanes, cross intersections or turn left/right. It is assumed that each vehicle knows the average transmission range, its position and velocity information that are transmitted using hello messages. The network is fully connected and each node can overhear the transmission situation of neighbouring ones. The OBU on each node is used to detect traffic, accidents, or other unforeseen situations and subsequent broadcasting. The additional parameters applied to safety services are as follows:

Mini- Slot - Length of a mini slot is set $T=2 d+t_{s}$ where d is the maximum signal propagation delay in the transmission range $\mathrm{R}$, and $\mathrm{t}_{\mathrm{s}}$ are the radio switch delay between reception and transmission modes [19].

BIFS - Broadcast Inter frame Space [20] is the time delay for which the emergency message sender waits after completing back off to sense an idle wireless channel. This is done to avoid interrupting ongoing Request To Send (RTS)/ Clear To Send (CTS)/ DATA/ ACK handshakes of neighbouring nodes and guarantee priority of messages. The length of BIFS $\left(\mathrm{T}_{\mathrm{B}}\right)$ needs to satisfy the condition: $T_{S}<T_{B}<T_{D}$ where, $T_{\mathrm{S}}$ is Short Inter frame Space and $T_{D}$ is Distributed Inter frame Space. DIFS is the time a node waits on sensing the channel to be idle before sending an RTS. SIFS is the time a node waits post receival of RTS to send the CTS.

Mini- CW - Mini-Contention Window (mini-CW) [21] is needed to avoid emergency message collisions when multiple sender nodes access the wireless channel asynchronously.

$$
\text { Mini }-C W=\frac{T_{D}-T_{B}}{T}
$$

In addition, OBUs make use of Global Positioning System (GPS) to acquire the position information of the vehicle. A digital map with the position information of road structures like intersections and multi-lanes is also available for each OBU.

\section{PROPOSED PROTOCOL 4.1 Protocol Overview}

The main advantages of BSPBR over conventional approach comes from two aspects namely, the use of backbone vehicles to forward broadcast data packets and a novel iterative partition transmission scheme. The backbone vehicles are updated periodically based on the topology information acquired through hello messages. The vehicle velocity, vehicle condition on the driving direction and channel quality are jointly taken into account for selection by using a fuzzy logic algorithm. These factors are calculated for all nodes in the transmission range to establish a database. The next remote single forwarder node is selected using iterative partitions and mini- slots that lower the transmission delay and reduces message redundancy. In case of more than one distinct node, the likeliness of nodes to be the backbone node is compared. Three broadcast schemes such as bi- directional broadcast, multi- directional broadcast and directional broadcast techniques are applied to the selected node. 


\subsection{Selection of Backbone Vehicles}

The protocol identifies a backbone node as the source node. It considers the vehicle velocity, the number of neighbour vehicles driving to the same direction and channel quality for selecting the backbone. The main concept is to use relatively slower vehicles. For a two-way road, the number of vehicles driving to one direction can be significantly larger than those moving in the other direction making it imperative to include directions. Channel condition is considered due to its dependence on the transmission device, antenna height and surrounding environment for different vehicles. Varying road segments and VANET scenarios make it difficult to derive a simple mathematical model. For the sake of flexibility, a fuzzy logic is used to jointly consider these metrics.

\subsection{Evaluation Based on Fuzzy Logic}

Fuzzy logic [22] can process approximate data by using nonnumeric linguistic variables to express facts. They are defined to convert numerical values to a fuzzy value. Rules are defined to process these and obtain the final fuzzy value. Using these each node evaluates its neighbours in close vicinity to determine the backbone node.

\subsubsection{Procedure}

The following procedure is defined to determine the competency value for each node:

- Calculation of multiple factors: The Velocity Factor, Directional Traffic Condition Factor and Channel Quality Factor are calculated for all nodes in the transmission range $\mathrm{R}$.

- Fuzzification: Predetermined linguistic variables and membership functions are used to convert these factors into fuzzy values.

- Mapping and combination of IF/THEN rules: Fuzzy values are mapped to IF/THEN rules and combined to get the final rank of the node.

\subsubsection{Calculation of Multiple Factors}

Upon reception of a hello message from a neighbour node X, node $\mathrm{S}$ calculates the following factors:

\section{- Velocity Factor (VF):}

Node $\mathrm{S}$ calculates the VF $(\mathrm{S}, \mathrm{X})$ using velocity $\mathrm{V}(\mathrm{X})$ of node $\mathrm{X}$ as: $V F(S, X)=\frac{|V(X)|-\min |V(Y)|}{\max |V(Y)|}$ where any node $\mathrm{Y} \in \mathrm{N}_{\mathrm{S}}$ i.e. neighbour set of node $\mathrm{S}$. This factor is updated with every hello interval using a weighted exponential as:

$$
V F(S, X) \leftarrow(1-m) * V F_{i-1}(S, X)+m * V F_{i}(S, X)
$$

Where $\mathrm{VF}_{\mathrm{i}-1}(\mathrm{~S}, \mathrm{X})$ and $\mathrm{VF}_{\mathrm{i}}(\mathrm{S}, \mathrm{X})$ denote the previous and current values of VF respectively. It is initialised to1. The coefficient $\mathrm{m}$ is set to 0.7 for best simulation results in most of the cases. It limits how quickly the evaluation value can change with respect to changing topology.

\section{- Directional Traffic Condition Factor (DTCF):}

Node $\mathrm{X}$ announces the number of neighbour vehicles $(\mathrm{C}(\mathrm{X})$ ) driving in the same direction using hello messages. DTCF is calculated as: $\operatorname{DTCF}(S, X)=\frac{C(X)}{\max C(Y)}$ where any node $\mathrm{Y} \in \mathrm{N}_{\mathrm{S}}$ neighbour set of node $S$. It indicates the vehicle density for the same direction. Higher value denotes increased suitability for being the backbone node. It is updated with every hello interval using the weighted exponential as:

$$
\begin{aligned}
\operatorname{DTCF}(S, X) & \leftarrow(1-m) * \operatorname{DTCF}_{i-1}(S, X)+m \\
& * \operatorname{DTCF}_{i}(S, X)
\end{aligned}
$$

\section{- Channel Quality Factor (CQF):}

It is expressed by the hello packet reception ratio. Each node maintains a counter to calculate the number of hello messages received from the neighbour nodes located within R. Since hello messages are sent with a predefined time interval (1 second by default), each node is able to calculate the reception ratio. The CQF is initialised to 0 and updated as:

$$
C Q F(S) \leftarrow(1-m) * C Q F_{i-1}(S, X)+m * C Q F_{i}(S, X)
$$

\subsubsection{Fuzzification}

The fuzzy membership functions of velocity factor, directional traffic condition factor and channel quality factor are defined in Figure 1. A node uses the velocity membership function to calculate the degree to which the velocity belongs \{Slow, Medium, Fast\}. Similarly the sender nodes calculate the degree of directional traffic condition \{Dense, Normal, Light\} and degree of channel quality factor \{Good, Medium, Bad\}.

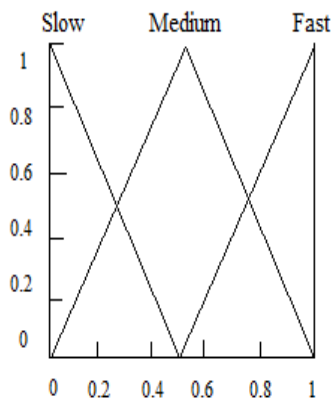

(a) Velocity Factor (VF)

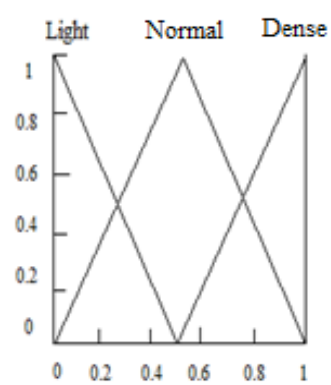

(b) Directional Traffic Condition Factor (DTCF)

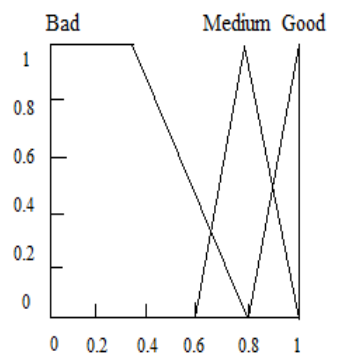

(c) Channel Quality Factor (CQF)

Figure 1.Fuzzy membership functions

\subsection{Mapping \& Combination of Rules}

Based on the fuzzy values of the velocity factor, directional traffic condition factor and channel quality factor, a node uses the IF/THEN rules as indicated in the Table 1 to calculate the rank of the vehicle as being the backbone node. The linguistic 
variables of the rank are defined as \{Perfect, Good, Acceptable, Not Preferred, Bad, Very Bad\}. IF Velocity is Slow, Traffic condition is Dense and Channel quality is Good, THEN Rank is Perfect. Since there can be multiple rules applying at the same time, the Min-Max method is used to combine evaluation results. The minimal value of the antecedent and maximal value of the consequents is used as the final degree.

Table 1.Rule Base

\begin{tabular}{|c|c|c|c|c|}
\hline RULE NO. & VELOCITY & TRAFFIC CONDITION & CHANNEL CONDITION & RANK \\
\hline 1 & Slow & Dense & Good & Perfect \\
\hline 2 & Slow & Dense & Medium & Good \\
\hline 3 & Slow & Dense & $\mathrm{Bad}$ & Unpreferable \\
\hline 4 & Slow & Normal & Good & Good \\
\hline 5 & Slow & Normal & Medium & Acceptable \\
\hline 6 & Slow & Normal & $\mathrm{Bad}$ & $\mathrm{Bad}$ \\
\hline 7 & Slow & Light & Good & Unpreferable \\
\hline 8 & Slow & Light & Medium & $\mathrm{Bad}$ \\
\hline 9 & Slow & Light & $\mathrm{Bad}$ & Very Bad \\
\hline 10 & Medium & Dense & Good & Good \\
\hline 11 & Medium & Dense & Medium & Acceptable \\
\hline 12 & Medium & Dense & $\mathrm{Bad}$ & $\mathrm{Bad}$ \\
\hline 13 & Medium & Normal & Good & Acceptable \\
\hline 14 & Medium & Normal & Medium & Unpreferable \\
\hline 15 & Medium & Normal & $\mathrm{Bad}$ & $\mathrm{Bad}$ \\
\hline 16 & Medium & Light & Good & $\mathrm{Bad}$ \\
\hline 17 & Medium & Light & Medium & $\mathrm{Bad}$ \\
\hline 18 & Medium & Light & $\mathrm{Bad}$ & Very Bad \\
\hline 19 & Fast & Dense & Good & Unpreferable \\
\hline 20 & Fast & Dense & Medium & $\mathrm{Bad}$ \\
\hline 21 & Fast & Dense & $\mathrm{Bad}$ & Very Bad \\
\hline 22 & Fast & Normal & Good & $\mathrm{Bad}$ \\
\hline 23 & Fast & Normal & Medium & $\mathrm{Bad}$ \\
\hline 24 & Fast & Normal & $\mathrm{Bad}$ & Very Bad \\
\hline 25 & Fast & Light & Good & $\mathrm{Bad}$ \\
\hline 26 & Fast & Light & Medium & Very Bad \\
\hline 27 & Fast & Light & $\mathrm{Bad}$ & Very Bad \\
\hline
\end{tabular}

\subsection{Transmission Scheme}

Several road scenarios like a one-way road, two-way road or intersections are possible which determine the broadcasting scheme to be employed. A backbone node with the highest rank is identified as the source node in the transmission range.

1) At the first hop, emergency message is bidirectionally broadcasted to neighbouring nodes if the source node is located on a straight road. Single relaying node is selected in either direction. It is multi-directionally broadcasted if the source node is located in an intersection area. A single relaying node is selected to forward a message in each road.
2) From the second hop onwards, the message is directionally broadcasted in the propagation direction using a single backbone relaying node.

\subsection{Bi-directional Broadcast}

Many immediate situations on road involve vehicles in two opposite directions and nearby ones need to be aware of any potential emergency. For example, the grey vehicle travels from left to right as shown in Figure 2. On detecting a dangerous event, it quickly notifies its neighbouring vehicles in the front direction (those going from left to right) and also those in the back direction (those going from right to left) within its transmission range $\mathrm{R}$. 


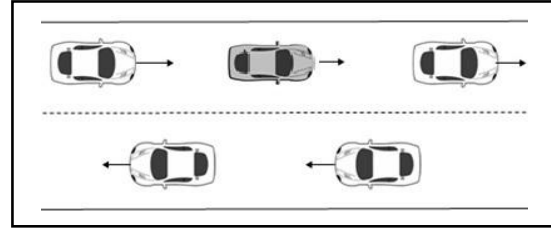

Figure 2.Bi-directional transmission

In order to disseminate the messages bi-directionally and reduce redundancy, the proposed protocol selects single forwarder backbone nodes in either directions of the source. On detecting an emergent event, the source node immediately broadcasts it into the medium access. As soon as the idle wireless channel is sensed, the source node randomly selects a mini-slot from mini-CW and starts the back off timer. If the channel keeps idle until the timer overflows, the message is directly broadcasted. Upon reception, the iterative candidate forwarding node selection process starts after SIFS interval for neighbouring nodes in the front and back direction simultaneously.

\section{1) Neighbouring nodes in the Front direction}

The first iteration lasts for two mini-slots. Transmission range $\mathrm{R}$ is partitioned into a Far Area (FA) and a Near Area (NA) in the front direction of the source node and the ratio of FA to $\mathrm{R}$ is denoted as $\alpha €(0,1)$ as shown in Figure 3(a). During the first mini-slot, neighbouring nodes in FA $((1-\alpha) *$ $R, R]$ send black-burst while nodes within NA $(0,(1-\alpha)]$ receive. In the second mini-slot of the iteration, NA is further partitioned in FA $\left((1-\alpha)^{2 R},(1-\alpha) * R\right]$ and NA $(0,(1-$ $\left.\alpha)^{2 R}\right]$ if all the residing nodes did not hear the black-burst initially as shown in Figure 3(b). Otherwise, NA is not further partitioned and all the nodes give up their opportunity to serve as the forwarding node. This is because some other farther node exists, which when transmits can be heard by all the previous nodes. A broadcast from any of the nodes in between can therefore be avoided to reduce total number of broadcasts. This farther node is determined by subsequently partitioning the FA into FA $\left(\left(1-\alpha^{2}\right) * R, R\right]$ and NA $((1-\alpha) *$ $\left.R,\left(1-\alpha^{2}\right) * R\right]$ if there are any neighbouring nodes within this FA as shown in Figure 3(b). During the second mini-slot

all nodes adjust their operations according to the new partition i.e. all new nodes in the new FA need to turn their radios into the transmitting mode while those in the new NA need to be in the receiving mode. This switch might cause a little delay during each iteration. Therefore, two min-slots are allocated for each iteration, one for transmitting and sensing and the other for radio switching. This ensures next iteration to operate correctly. The second iteration starts from the third mini-slot. A neighbouring node in FA sends the black-burst for one mini-slot while those in NA receive. In the next minislot, the FA or NA is further partitioned with the same principle as used in the first iteration. This process is continued until the iteration time reaches $\mathrm{N}$ as shown in Figure 3(c), the value of which is limited to the condition, where $N \leq \frac{T_{D}-T_{S}-T}{2 T}$. In the $\mathrm{N}^{\text {th }}$ iteration that lasts for three mini-slots, the neighbouring nodes in the FA send black-burst in the $2 \mathrm{~N}-1^{\text {th }}$ mini-slot and then successfully become the candidate forwarding nodes in the front direction. However if there are no nodes are located in the FA, neighbouring nodes in the farthest NA become the candidate forwarding nodes. In case of more than one node, the ranks of nodes to become the backbone node are compared and the highest one becomes the final forwarding node. In the last mini-slot, this node sends a black-burst message to reserve the wireless channel resources.
As a result, the remote nodes that do not locate within the coverage range of $\mathrm{R}$ of the source node will keep the channel idle for at least DIFS interval to avoid interference.

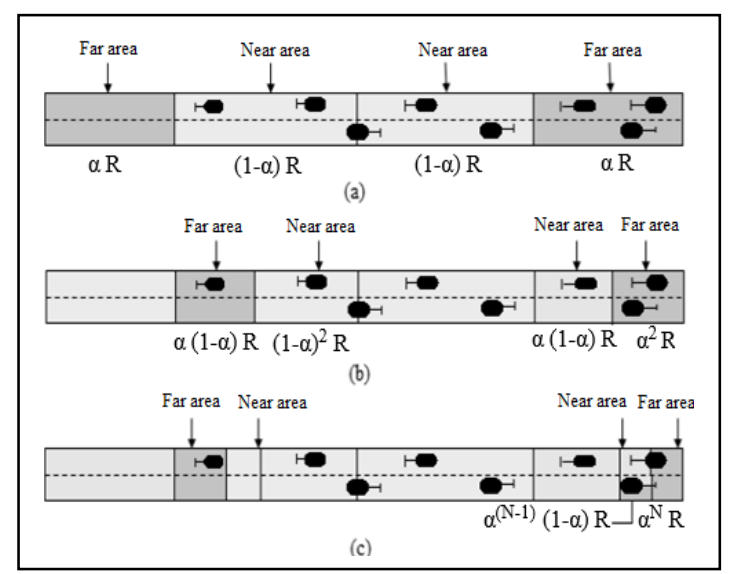

(a) First partition (b) Second partition (c) $\mathbf{N}^{\text {th }}$ partition

Figure 3.Partitions of transmission range $\mathbf{R}$

\section{2) Neighbouring nodes in the Back direction}

Neighbouring nodes in the back direction should not transit the black-burst messages simultaneously as that in the front direction to avoid interference in either directions of the source node. For example, as shown in Figure 3(a), the neighbouring nodes in the left NA may hear black-bursts from those within the right NA in the first iteration consequently giving up the opportunity to serve as the candidate forwarding node. This prevents emergency message propagation in that direction. To address the same issue, the iteration process in both the directions are conducted alternately as shown in Figure 4. The nodes in the front direction sense or transmit black-burst in the first iteration and switch radios in the second (Figure 4(a)). The process is reversed in the back direction (Figure 4(b)). In addition, the same partition principle as in the front direction is utilized to obtain the FA and NA in each iteration in the back direction. As a result, the iteration process is performed simultaneously in opposite directions of the source node until the candidate forwarding nodes are successfully selected in either direction.

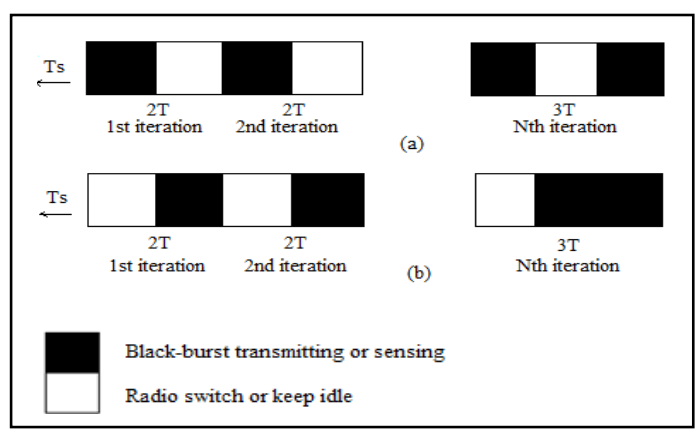

Figure 4.Actions of neighbouring nodes in opposite directions (a) Neighbouring nodes in the front direction (b) Neighbouring nodes in the backward direction

After $\mathrm{N}$ iterations on sensing the wireless channel idle for SIFS interval, a candidate forwarding node randomly selects a mini-slot from the contention window $(\mathrm{CW})$ and starts the back off process where, $C W=\frac{T_{D}-T_{S}}{T}$. If the wireless channel keeps idle until the back off timer overflows, the forwarding node sends and RTS. All other nodes give up their opportunity to serve as a forwarding node. The RTS/CTS 
handshake is established for directional broadcast along the propagation direction on a road.

\subsection{Multi-directional Broadcast}

GPS localization and digital map enable all nodes to identify an intersection area. When an emergency occurs at an intersection area, BSPBR conducts multi-directional broadcast at the first hop to guarantee message propagation along all road branches. It follows the same three steps as in bidirectional. A more complex candidate forwarding node selection process is conducted due to involvement of nodes from all directions simultaneously. The protocol needs to eliminate the black-burst interference among neighbouring nodes in different directions. Within an intersecting area, it is possible to prevent the black-burst transmitted by the neighbouring nodes on one road from covering its intersecting road as long as it needs to reach the nodes in the opposite direction of the intersection. For example, $\mathrm{S}$ is the source node and $\mathrm{X}, \mathrm{Y}, \mathrm{Z}$ and $\mathrm{C}$ are four neighbouring nodes in different directions of the intersection $\mathrm{O}$ as shown in Figure 5. Blackburst messages transmitted from $\mathrm{C}$ to source node $\mathrm{S}$ other neighbouring nodes covers nodes on both the intersecting nodes. Multi-directional broadcast handles this black-burst interference using a novel approach to regulate transmissions.

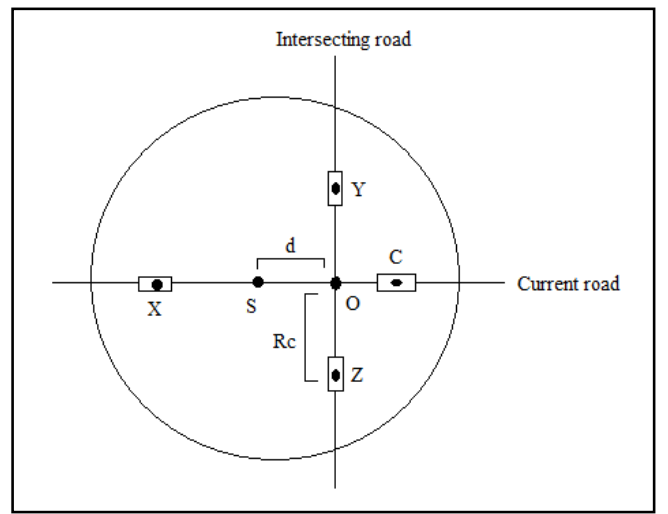

Figure 5.Multi-directional broadcast at an intersection

On receiving the broadcast emergency message, a neighbouring node decides how to transmit or sense the blackburst depending on the road it is situated on. The road where the source node is located is called the current road and the one that intersects it is the intersection road. With the position information of the source node given by the digital map, any neighbouring node can identify whether it is located on the current road or intersecting road and hence start the candidate selection process.

\section{1) Neighbouring nodes on the current node}

For neighbouring nodes on the current node such as $\mathrm{X}$ and $\mathrm{C}$ as shown in Figure 5, the iteration process is similar to that in bi-directional broadcast. In the first mini-slot of any iteration the neighbouring nodes in the front direction transmit or sense black-burst, while those in the back direction may switch radios or keep idle. In the next mini-slot of the iteration, the operations are reversed. After $\mathrm{N}$ iterations, candidate forwarding node in either direction of the current node is selected successfully. This node then keeps idle for $2 \mathrm{NT}$ interval and thereafter transmits black-burst for one mini-slot to reserve the wireless channel for following transmission as shown in Figure 6(a).

\section{2) Neighbouring nodes on the Intersecting road}

The iteration process of nodes on the intersecting road such as $\mathrm{Y}$ and $\mathrm{Z}$ as shown in Figure 5 is much different than those on the current road. Before the iteration process, the node needs to compute two distances: (i) distance from the source node to the intersection d; and (ii) covered length of the intersecting road by the source node, half of which is denoted as $R_{c}=$ $\sqrt{R^{2}-d^{2}}$ as shown in Figure 5. The first iteration starts at $T_{S}+(2 N+1) * T$ interval on receiving the broadcast message as shown in Figure $6(\mathrm{~b}) . \mathrm{R}_{\mathrm{C}}$ is partitioned into FA $\left((1-\alpha) * R_{c}, R_{c}\right]$ and NA $\left(0,(1-\alpha) * R_{c}\right]$ in each direction of the intersecting road. In the first mini-slot of the iteration, the neighbouring nodes in one direction transmit or sense black- burst and those in the other direction may switch their radios or keep idle. In the second mini-slot, their operations are reversed. Following this, the same principles as those in bi-directional broadcast are adopted to obtain the FA and NA in each direction. The above procedure is repeated for $\mathrm{N}$ iterations to obtain the candidate forwarding nodes in each direction. The neighbouring nodes on both the roads perform the iteration process alternately to eliminate black-burst interference. The above operations help select a single forwarding node in each direction using multi-directional broadcast followed by directional propagation.

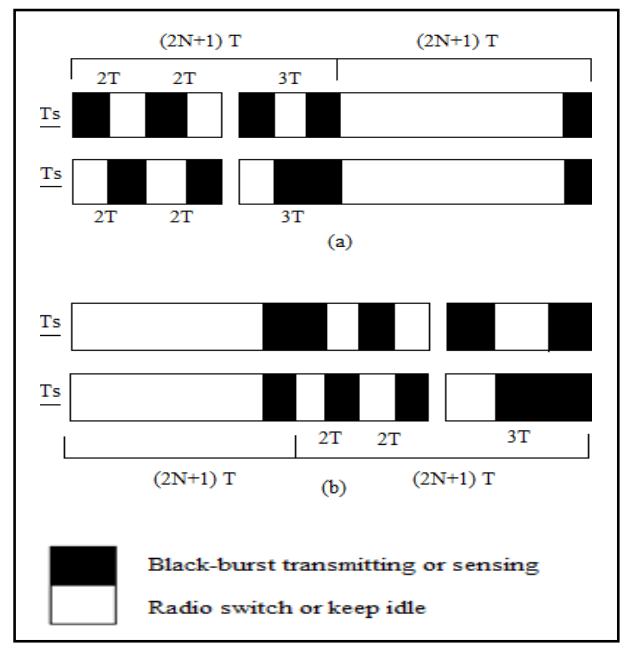

Figure 6.Iteration process on different roads (a) Iteration on current road (b) Iteration on intersecting road

\subsection{Directional Broadcast}

From the second hop, the emergency message is directionally broadcast as long as the forwarding node is not located in an intersecting area. RTS/CTS handshake is utilized to solve the hidden terminal problem.

In BSPBR, backbone node is selected to serve as the source node for all emergency messages. Bi-directional broadcast and multi-directional broadcast is utilized at the first hop and the forwarding node selection scheme is conducted simultaneously in different road directions. This greatly reduces message redundancy and decreases transmission hops for emergency messages thereby lowering transmission delay. The directional broadcast uses RTS/CTS handshake to improve message reliability in the single direction of propagation. This ensures seamless coverage of the target area in urban environment. In summary, the emergency message dissemination strategies are described as Algorithm 1. 


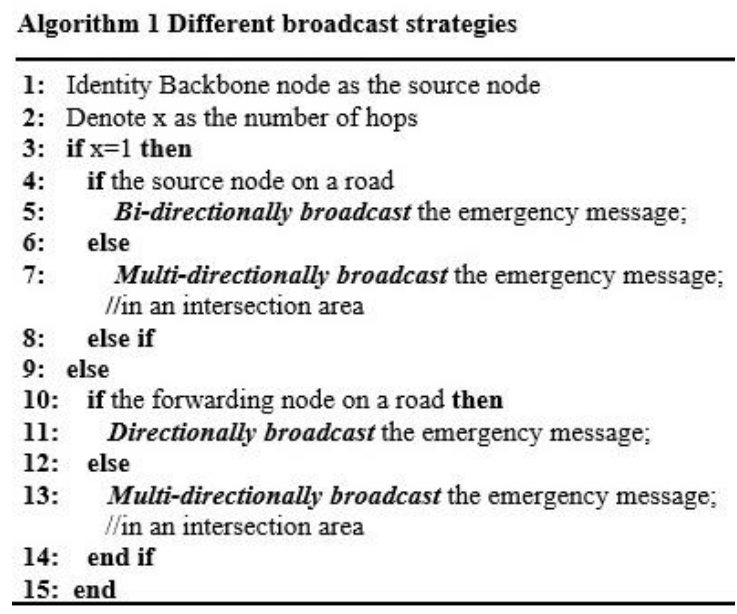

\section{THEORETICAL ANALYSIS}

Efficiency of BSPBR is checked against varying number of nodes for critical emergency parameters like throughput, packet delivery fraction and end-to-end delay. The following assumptions are made to make it tractable:

1. Vehicles are distributed on an M-lane road with varying large traffic flows.

2. An emergency situation occurs either on a road or within an intersection area. From amongst the vehicles that first detect the situation, the one with the highest rank for backbone node is chosen as the source node to initiate message dissemination.

3. Packets are successfully received as long as there is no packet collision within the transmission range $\mathrm{R}$. Packet loss due to channel error is not considered.

Throughput-- It is the maximum data rate that a system can achieve in a given time period typically measured in bits per second (bps) [23]. It is the sum of sizes (bits) or number (packets) of generated/sent/forwarded/received packets calculated at every time interval and divided by its length.

End-to-End Delay-- It refers to the time taken for a packet to be transmitted across a network from source to destination [24]. The transmitted packet needs to be identified at both the synchronised points to avoid packet loss or packet reordering.

$$
D_{\text {end-end }}=n *\left(d_{\text {trans }}+d_{\text {prop }}+d_{\text {proc }}+d_{\text {queue }}\right)
$$

Where, $D_{\text {end-end }}$ is the end-to-end delay, $d_{\text {trans }}$ is the transmission delay, dprop is the propagation delay, $\mathrm{d}_{\text {proc }}$ is the processing delay, $\mathrm{d}_{\text {queue }}$ is the queuing delay and $\mathrm{n}$ is the number of links.

Packet Delivery Fraction-- It is the ratio of the data packets received by the destination to those generated by the source [20]. Mathematically, it can be defined as, $P D F=\frac{S 1}{S 2}$ where, $\mathrm{S} 1$ is the sum of data packets received by each destination and $\mathrm{S} 2$ is the sum of data packets generated by each source.

\section{SIMULATION RESULTS}

In this section, the proposed BSPBR protocol has been implemented in Network Simulator (NS-2.35) [25] and its performance evaluated in terms of throughput, end-to-end delay and packet delivery fraction. It identifies a source backbone node and uses multi-dimensional broadcast strategies to determine subsequent forwarding nodes. These sequentially select the next hop relaying node in all directions. The conventional IEEE $802.11 \mathrm{~g}$ is used as the base MAC protocol. The simulated urban network adopts the Manhattan mobility model for a number of horizontal and vertical nodes. Vehicles are randomly distributed in different two lane roads. Minimum inter-vehicle distance between two neighbours is $20 \mathrm{~m}$, which means that the maximum vehicle density on a lane $V_{\text {max }}=\frac{1}{20}$ vehicles $/ \mathrm{m}$. The length of a road segment between two neighbouring intersections is set to $600 \mathrm{~m}$. Same simulation is conducted 10 times using the proposed protocol by varying number of nodes on intervals of 10 and average results are calculated. Detailed performance settings used in the simulations are tabulated in Table 2. The factors are evaluated on the basis of variation with number of nodes.

Table 2.Parameters in simulations

\begin{tabular}{|l|l|}
\hline PARAMETER & VALUE \\
\hline Routing protocol & AODV \\
\hline Simulation time & 200 seconds \\
\hline Number of Nodes & $10,20,30,40,50,60,70,80,90,100$ \\
\hline Simulation area & $600 \times 600$ \\
\hline Antenna & Omni-directional \\
\hline MAC & IEEE $802.11 \mathrm{~g}$ \\
\hline Traffic & CBR \\
\hline Packet size & 512 bytes \\
\hline Channel type & Wireless \\
\hline Propagation & Two way ground reflection \\
\hline
\end{tabular}

\subsection{Throughput}

Figure 7 illustrates the performance of the network by varying number of nodes and pause time. It is directly related to the packet drops caused by network congestion and lack of route. Since most of the routing protocols (proactive or reactive) try to keep the latter low by being responsive to topology changes, the network congestion drops become the dominant factor when judging throughput performance. A remarkable improvement is observed showing greater efficiency of the proposed protocol to that of general wireless urban scenarios as the number of nodes increases beyond 30. Two protocols coincide at 50 nodes, post which BSPBR significantly takes over AODV. As the number of nodes or traffic flow is increased, throughput also increases proportionally.

\subsection{End-to-End Delay}

For time critical safety services, the end-to-end delay of emergency messages is the most important performance metric. For a certain set of conditions, it is evaluated by varying the number of nodes and noting the values at each node point. In Figure 8, it can be observed that the end-to-end delay does not have a remarkable change in the simulation. At a certain node point in the graph, the network shows slight improvement in the results for the proposed protocol.

\subsection{Packet Delivery Fraction (PDF)}

Figure 9 gives the result of packet delivery fraction vs. Number of nodes, which shows that the proposed approach can effectively improve packet delivery fraction in case of high resource contention. The proposed approach begins to outperform the general approach when number of source nodes becomes larger than 40 . 


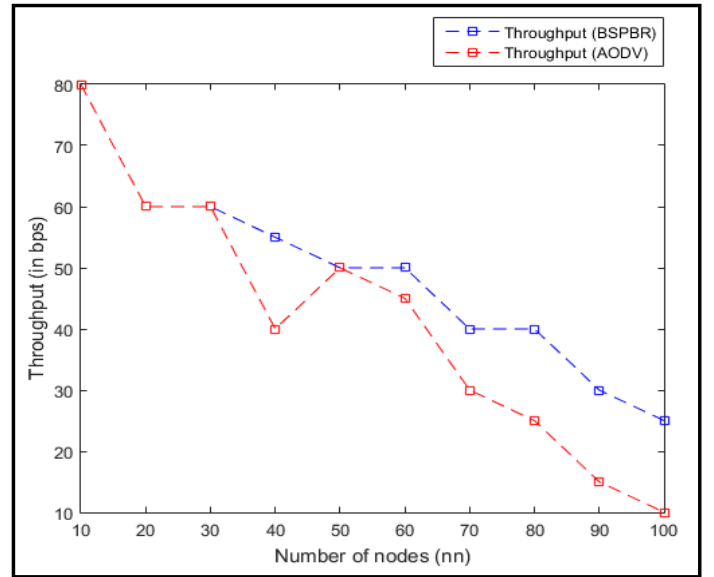

Figure 7.Throughput analysis for BSPBR protocol

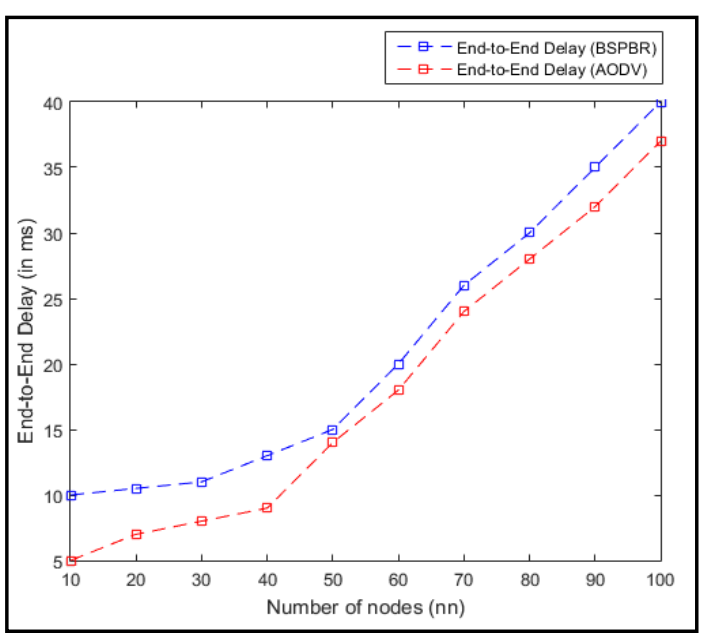

Figure 8.End-to-end delay analysis for BSPBR protocol

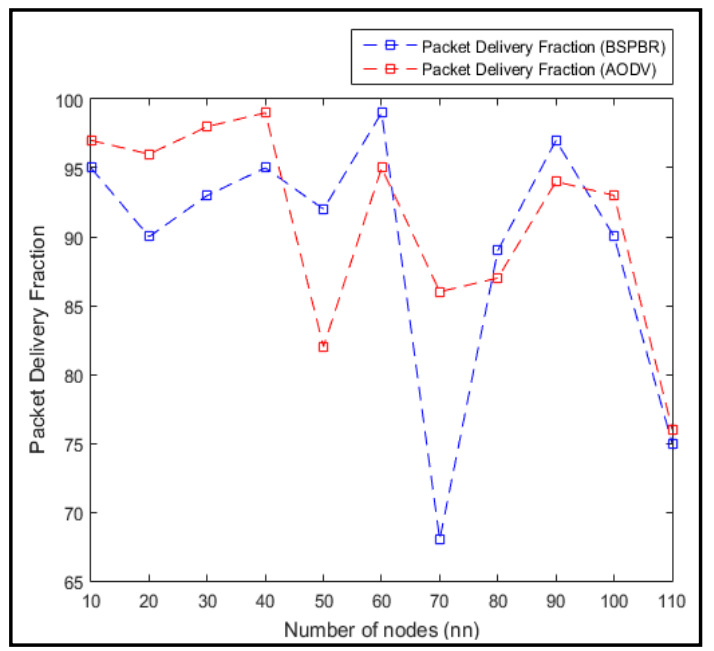

Figure 9.PDF analysis for BSPBR protocol

\section{CONCLUSION}

As an important component of the ITS, VANETs have attracted considerable attention. Event-driven emergency message dissemination with high reliability and low delay is vital to ensure traffic safety and improve traffic efficiency in urban environments. In this paper, an efficient multi-hop broadcast protocol, BSPBR has been proposed which employs backbone source nodes and positional forwarding nodes. The protocol generates dynamic vehicle backbone by taking into account vehicle movement and link quality based on a fuzzy logic. It greatly reduces the number of source nodes resulting in a shorter MAC layer contention time. The first hop utilizes a bi-directional or multi-directional broadcast scheme to select the most efficient forwarder nodes in all directions. Directional broadcast is adopted for message propagation along different directions. It disseminates messages quickly with less redundancy and enhanced reliability. The proposed protocol can provide a lightweight and efficient solution for data dissemination in VANETs. Theoretical analysis and simulation results confirmed the advantage of the proposal over other general existing alternatives.

\section{REFERENCES}

[1] Taleb, T., Sakhae, E., Jamalipour, A., Hashimoto, K., Kato, N., and Nemoto, Y. 2007, "A Stable Routing Protocol to Support ITS Services in VANET Networks", IEEE Transactions on Vehicular Technology, 56 (Nov. 2007), 3337-3347

[2] He., J., Cai, L., Cheng, P., and Pan, J. 2016, "Delay Minimization for Data Dissemination in Large-scale VANETs with Buses and Taxis", IEEE Transactions on Mobile Computing, 15 (Aug. 2016), 1939-1950

[3] Akabane, A., Madeira, E., and Villas, L. 2015, "A Suitable Broadcast Protocol under Different Traffic Patterns for Urban Scenario", IEEE Latin America Transactions, 13 (Jan. 2015), 222-227

[4] Ros, F., Ruiz, P., and Stojmenovic, I. 2009, "Reliable and efficient broadcasting in vehicular ad hoc networks", Proc. IEEE VTC Spring, 1-5

[5] Hayat, U., Iqbal, R., and Diab, J. 2016, "Eliminating Broadcast Storming in Vehicular Ad-hoc Networks", International Journal of Advanced Computer Science and Applications (IJACSA), 7 (Jan. 2016), 348-354

[6] Wisitpongphan, N., Tonguz, O., Parikh, J., Mudalige, P., Bai, F., and Sadekar, V. 2007, "Broadcast storm mitigation techniques in vehicular ad hoc networks", IEEE Wireless Communications, 14 (Dec. 2007), 84-94

[7] Bi, Y., Liu, K., Cai, L., Shen, X., and Zhao, H. 2009, "A multi-channel token ring protocol for QoS provisioning in inter-vehicle communication", IEEE Transactions Wireless Communications, 8 (Nov. 2009), 5621-5631

[8] Yang, C., and Lo, S. 2010, "Street broadcast with smart relay for emergency messages in VANET", Proc. IEEE $24^{\text {th }}$ International Conference on Advance Information and Network Applications Workshops, 323-328

[9] Ma, X., Zhang, Z., Yin, X., and Trivedi, K. 2012, "Design and analysis of a robust broadcast scheme for VANET safety related services", IEEE Transactions on Vehicular Technology, 61 (Jan, 2012), 46-61

[10] Suthaputchakun, C.,Dianati, M., and Sun, Z. 2014, "Trinary partitioned black-burst based broadcast protocol for time-critical emergency message dissemination in VANETs", IEEE Transactions on Vehicular Technology, 63 (Jul. 2014), 2926-2940

[11] Bi, Y., Cai, L., Shen, X., and Zhao, H. 2010, "Efficient and reliable broadcast in intervehicle communications networks: A cross-layer approach", IEEE Transactions on Vehicular Technology, 59 (Jun. 2010), 2404-2417

[12] Mylonas, Y., Lestas, M., Pistillides, A., Ioannou, P., and Papadopoulou, V. 2015, "Speed Adaptive Probabilistic Flooding for Vehicular Ad Hoc Networks", IEEE Transactions on Vehicular Technology, 64 (May 2015), 1973-1990

[13] Wu, L., Nie, L., Fan, J., He, Y., Liu, Q., and Wu, D. 2017, “An Efficient Multi-hop Broadcast Protocol for 
Emergency Messages Dissemination in VANETs", 26 (Mar. 2017), 614-623

[14] Martinez, F., Fogue, M., Coll, M., Cano, J., Calafate, C., and Manzoni, P. 2010, "Evaluating the Impact of a Novel Warning Message Dissemination Scheme for VANETs Using Real City Maps”, Proc. IFIP Networking, 265-276

[15] Fogue, M., Garrido, P., Martinez, F., Cano, J., Calafete, C., and Manzomi, P. 2013, "An Adaptive System Based on Roadmap Profiling to Enhance Warning Message Dissemination in VANETs", IEEE/ACM Transactions on Networking, 21 (Jun. 2013, 883-895

[16] Korkmaz, G., Ekici, E., and Ozguner, F. 2007, "Blackburst based multihop broadcast protocols for vehicular networks", IEEE Transactions on Vehicular Technology, 56 (Sept. 2007), 3159-316

[17] Sahoo, J., Wu, E., Sahu, P., and Gerla, M. 2011, "Binarypartition-assisted MAC-layer broadcast for emergency message dissemination in VANETs", IEEE J. Sel. Areas Communications, 12 (Sept. 2011), 757-770

[18] Zhu, W., Gao, D., Foh, C., Zhang, H., and Chao, H. 2017, "Reliable emergency message dissemination protocol for urban internet of vehicles", IET Communications, 11 (Jun. 2017), 1275-1281

[19] Cheng, X., Yang, L., and Shen, X. 2015, "D2D for intelligent transportation systems: A feasibility study",
IEEE Transactions on Intelligent Transportation System, 16 (Aug. 2015), 1784-1793

[20] Forouzan, B., and Fegan, S. 2006, Data Communication and Networking

[21] Yng, Q., Xing, S., Xia, W., and Shen, L. 2015, "Modelling and performance analysis of dynamic contention window scheme for periodic broadcast in vehicular ad hoc networks", IET Communications, 9 (Jul. 2015), 1347-1354

[22] Klr, G., Clair, U., and BO. Y. 1995, Fuzzy set theory: foundations and applications, Prentice-Hall

[23] Wang, M., Shan, H., Luan, T., Lu, N., Zhang, R., Shen, X., and Bai, F. 2015, "Asymptotic Throughput Capacity Analysis of VANETs Exploiting Mobility Diversity", IEEE Transactions on Vehicular Technology, 64 (Sept. 2015), 4187-4202

[24] He, J., Cai, L., Cheng, P., and Pan, J. 2017, "Delay Analysis and Routing for Two-Dimensional VANETs Using Carry-and Forward Mechanism", IEEE Transactions on Mobile Computing, 16 (Jul. 2017), 1830-1841

[25] The Network Simulator-ns 2.35 [Online], Available: http://www.isi.edu/nsnam/ns/ 